\title{
O HIPOTÁLAMO DORSOMEDIAL E A ORGANIZAÇÃO DA RESPOSTA CARDIOVASCULAR AO ESTRESSE EMOCIONAL: UMA PERSPECTIVA FUNCIONAL
}

\author{
THE DORSOMEDIAL HYPOTHALAMUS AND THE ORGANIZATION OF THE CARDIOVASCULAR \\ RESPONSE TO EMOTIONAL STRESS: A FUNCTIONAL PERSPECTIVE
}

Marco AP Fontes ${ }^{1}$, Rodrigo CA Menezes², Daniel C Villela³ ${ }^{3}$ Luiz G da Silva Jr².

${ }^{1}$ Docente. ${ }^{2}$ Doutorando. ${ }^{3}$ Mestrando. Laboratório de Hipertensão. Departamento de Fisiologia e Biofísica. Instituto de Ciências Biológicas - UFMG.

Correspondência: Dr. Marco AP Fontes

Laboratório de Hipertensão. Departamento de Fisiologia e Biofísica - ICB. UFMG. CEP: 31270-901 - Belo Horizonte, MG

Tel: 3134992953 / Fax: 3134992924 / Email: peliky@mono.icb.ufmg.br

Fontes MAP, Menezes RCA, Villela DC, Silva Jr LG. O hipotálamo dorsomedial e a organização da resposta cardiovascular ao estresse emocional: uma perspectiva funcional. Medicina (Ribeirão Preto) 2006; 39 (1): 13-20.

RESUMO: O estresse emocional resulta em ativação de vias específicas do sistema nervoso central, que produzem respostas autonômicas, comportamentais e endócrinas. Sabe-se que situações de estresse recorrentes ou prolongadas podem resultar em vários estados patológicos, como por exemplo, a hipertensão arterial.

O Hipotálamo tem papel fundamental na integração das respostas fisiológicas ao estresse emocional. Particularmente, estudos têm mostrado que um núcleo específico do hipotálamo, o hipotálamo dorsomedial (DMH), é um componente fundamental das vias centrais mediadoras das respostas cardiovasculares ao estresse emocional. A inibição dos neurônios dessa área reduz os aumentos de freqüência cardíaca e de pressão arterial em ratos quando submetidos à situações de estresse emocional. Ao contrário, a ativação farmacológica dos neurônios do DMH produz aumento na frequência cardíaca, pressão arterial, hormônio adrenocorticotrópico (ACTH), atividade locomotora e na atividade simpática para diversos leitos vasculares. A similaridade dessa resposta com aquela produzida durante a situação real de estresse emocional sugere que esta área é fundamental na integração da resposta fisiológica ao estresse.

A presente revisão tem como objetivo mostrar, através de resultados de estudos recentes, as vias centrais utilizadas pelo DMH na organização da resposta cardiovascular ao estresse emocional.

Descritores: Hipotálamo Dorsomedial. Estresse Emocional. Sistema Cardiovascular. Vias Centrais Descendentes.

\section{1- INTRODUÇÃO}

O estresse emocional resulta em ativação de vias específicas do sistema nervoso central, que vão produzir respostas comportamentais, autonômicas e endócrinas. É de consenso geral que o surgimento destes circuitos específicos durante à evolução, permitiu ao organismo responder rápida e estrategicamen- te às ameaças do ambiente ${ }^{1}$, e que a ativação destes de maneira integrada facilita a sobrevivência das diferentes espécies. Por exemplo, particularmente em relação ao sistema cardiovascular, as reações de ataque ou fuga são acompanhadas de aumento na pressão arterial, frequência cardíaca e alteração do fluxo sanguíneo para os leitos muscular, cutâneo, renal e esplâncnico ${ }^{2}$. Essas alterações ocorrem em grande 
parte, através da ativação do sistema nervoso simpático com a finalidade de melhorar a performance do indivíduo numa situação de emergência ${ }^{1,2}$.

Em condições normais, os parâmetros cardiovasculares alterados pelo estresse emocional retornam rapidamente ao estado basal ao final do estímulo estressante, devido aos mecanismos fisiológicos de auto-regulação ${ }^{3}$. No entanto, situações de estresse repetidas ou prolongadas podem resultar em vários estados patológicos, como a hipertensão arterial, arritmias cardíacas, infarto do miocárdio e até mesmo a morte súbita ${ }^{3,4,5}$. Interessantemente, estudos recentes em humanos mostram que um aumento sustentado da pressão arterial pode ocorrer na presença de situações de estresse exacerbado ${ }^{6,7}$. Além disso, outros distúrbios do estresse, como o transtorno do pânico, apresentam uma correlação positiva com a hipertensão essencial ${ }^{8}$. É importante ressaltar que estudos em animais corroboram os achados em humanos, por exemplo, a ativação repetida da resposta cardiovascular induzida pelo estresse emocional em ratos produz hipertensão arterial sustentada ${ }^{9}$. Desta forma, e particularmente nesse caso, o modelo experimental torna-se extremamente útil como ferramenta de investigação sobre os mecanismos centrais envolvidos na fisiologia e fisiopatologia do sistema cardiovascular em consequência à exposição ao estresse emocional.

\section{2- LOCALIZAÇÃO ANATÔMICA}

O DMH está situado adjacente ao terceiro ventrículo, caudal ao núcleo paraventricular do hipotálamo, dorsal ao núcleo ventromedial e ventral à zona incerta (Figura 1). Suas bordas laterais e caudais são pouco distintas. Lateralmente, este é circundado pela região perifornical da área hipotalâmica lateral, com os limites do seu pólo caudal terminando no núcleo periventricular posterior ${ }^{10}$. O DMH é ainda subdividido em duas porções distintas; uma porção difusa e uma porção compacta ${ }^{11}$, sendo esta última claramente delimitada no polo posterior do DMH. Interessantemente, estudos indicam que a porção compacta do $\mathrm{DMH}$ é a estrutura mais responsiva do $\mathrm{DMH}$ à microinjeção de aminoácidos excitatórios ${ }^{12}$ ou de antagonistas do receptor GABA $_{\mathrm{A}}{ }^{12 / 16}$ (Figura 2 e Diagrama à direita). Possivelmente, como será mencionado nos tópicos subsequentes, esta seria a região crítica onde neurônios desta área enviariam projeções axonais para outros núcleos do sistema nervoso central envolvidos com o controle de funções cardiovasculares.

Estudos funcionais envolvendo hipotálamo em humanos são raros, no entanto a comparação estrutural do hipotálamo humano com a de outras espécies nos fornece uma referência importante, permitindo extrapolar achados fisiológicos em animais experimen-

Em função da estreita correlação entre estresse e doença cardiovascular, vários estudos têm sido realizados com o intuito de aprofundar o entendimento da organização central da resposta cardiovascular ao estresse emocional. A identificação de núcleos do sistema nervoso central (SNC), vias e os neurotransmissores envolvidos na organização dessa resposta pode, futuramente, possibilitar o desenvolvimentos de procedimentos mais adequados no tratamento desses distúrbios. A presente revisão tem por objetivo mostrar as vias centrais utilizadas por um núcleo hipotalâmico em particular, o hipotálamo dorsomedial $(\mathrm{DMH})$ na geração da resposta cardiovascular ao estresse emocional. Anteriormente às considerações funcionais do $\mathrm{DMH}$, faremos uma descrição da localização anatômica deste núcleo.

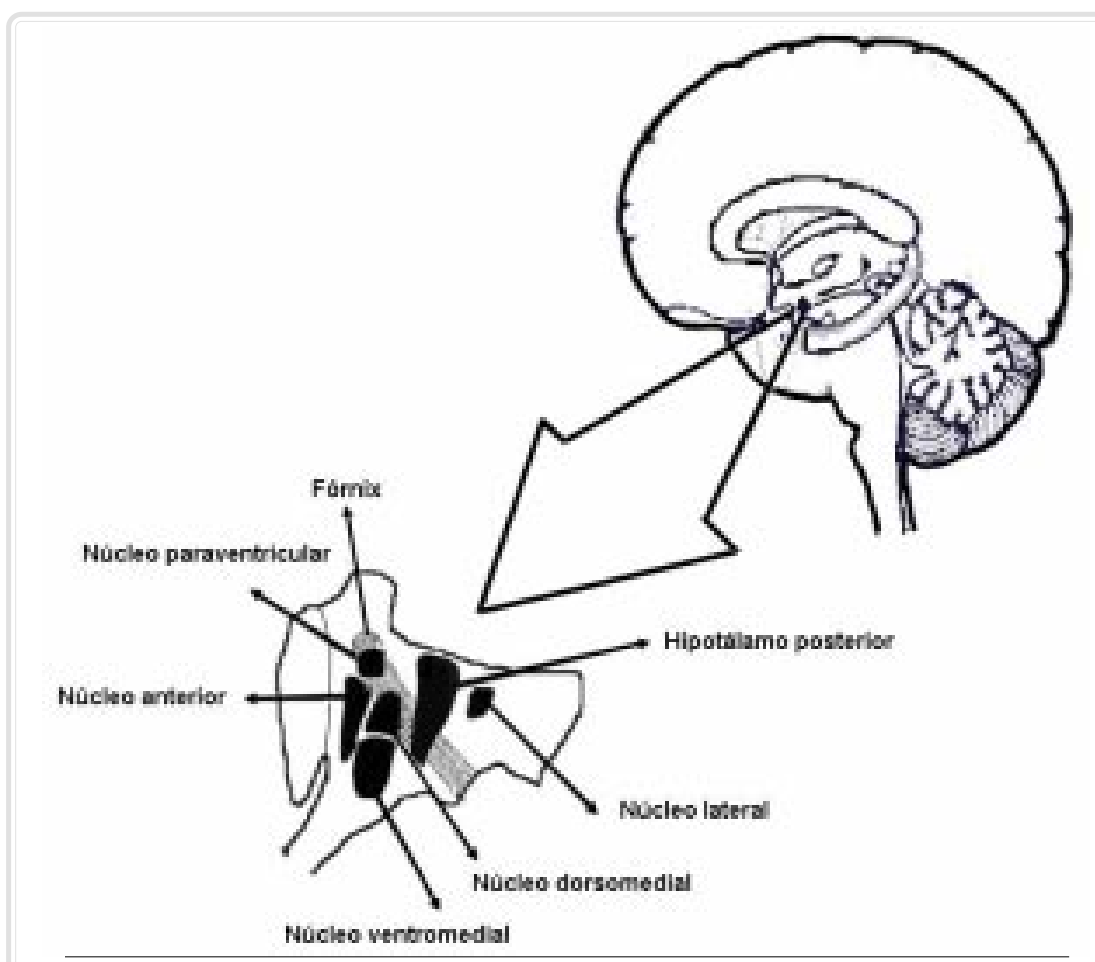

Figura 1: Diagrama de corte sagital do cérebro humano mostrando os principais núcleos hipotalâmicos. 


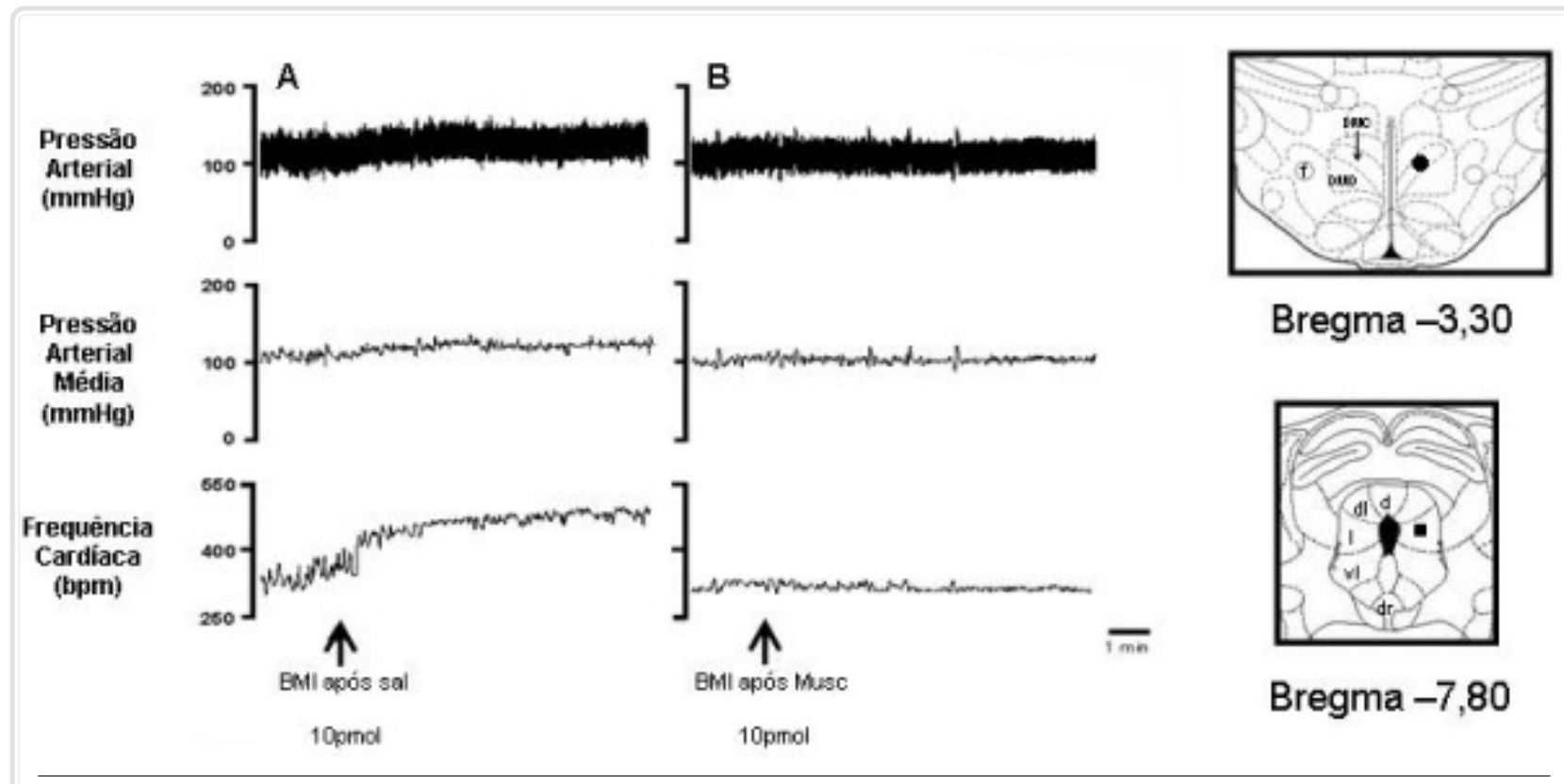

Figura 2: Esquerda, registro de um experimento mostrando a resposta cardiovascular produzida pela ativação do DMH antes (A) e após (B) a inibição dos neurônios da l/dIPAG. Direita, esquema das secções coronais do cérebro do rato mostrando os sítios de injeção no DMH e PAG. BMI, bicuculina methiodide; DMC, porção compacta do hipotálamo dorsomedial; DMD, porção difusa do hipotálamo dorsomedial; d, PAG dorsal; dl, PAG dorsolateral; I, PAG lateral; vl, PAG ventrolateral; dr, núcleo dorsal da rafe. Adaptado de Da Silva et al., $2003^{14}$.

tais para humanos. Nesse sentido, tem sido demonstrado que o hipotálamo humano é mais similar ao hipotálamo do rato do que se pensava anteriormente ${ }^{17}$. Em relação ao DMH, Koutcherov et al. ${ }^{17,18}$ utilizando marcadores químicos, mostraram que o DMH humano é similar ao DMH do macaco e do rato. No feto humano, o DMH é claramente definido já na décima semana de gestação ${ }^{17}$. De maneira interessante, os sintomas que acompanham os ataques de pânico em humanos são caracterizados por disfunção autonômica, como importante taquicardia ${ }^{19,20}$ e aumento da atividade simpática para o coração ${ }^{21}$ - alterações similares às observadas durante a estimulação do DMH em ratos. Adicionalmente, a disfunção crônica de GABA no DMH de ratos resulta em um modelo experimental de desordem do pânico, ao menos no que diz respeito aos aspectos cardiovasculares e comportamentais ${ }^{22}$. Existem evidências de que o padrão autonômico da resposta emocional ao estresse parece ser o mesmo pelo menos no rato ${ }^{4}$, macaco ${ }^{23}$ e humanos $^{24}$. Esta observação, somada aos achados anatômicos descritos acima, nos permite especular que as vias envolvidas na resposta cardiovascular ao estresse produzidas pela ativação do DMH em animais experimentais podem, provavelmente, ser correspondentes em humanos.

\section{3- DMH: UMA ÁREA CRÍTICA NA MEDIA- ÇÃO DAS RESPOSTAS FISIOLÓGICAS AO ESTRESSE EMOCIONAL}

Nos últimos anos, estudos têm demonstrado que o DMH é um componente importante das vias centrais mediadoras da resposta cardiovascular e comportamental ao estresse emocional ${ }^{4,25}$. A microinjeção do agonista $\mathrm{GABA}_{\mathrm{A}}$ muscimol - um potente inibidor neuronal ${ }^{26}$ especificamente no $\mathrm{DMH}$ reduz de maneira importante a taquicardia e hipertensão em ratos quando submetidos a situações de estresse emocional $^{27}$. Ao contrário, a ativação de neurônios do DMH em ratos anestesiados através da microinjeção dos aminoácidos excitatórios NMDA e ácido kaínico ou pelo antagonista dos receptores $\mathrm{GABA}_{\mathrm{A}}$ bicuculina methiodide (BMI), produz um importante aumento na frequência cardíaca e uma elevação modesta da pressão arterial mediadas pela ativação do sistema nervoso simpático ${ }^{28,29}$. Adicionalmente, outros estudos em ratos acordados mostraram que a ativação do DMH pelos antagonistas GABAérgicos BMI e picrotoxina produz aumento de frequência cardíaca, pressão arterial e um padrão de comportamento similar ao observado em estados de ansiedade como a síndrome do 
pânico $22,30,31$. Esses resultados sugerem que o DMH possui um papel importante na regulação das respostas cardiovasculares e comportamentais ao estresse emocional $^{22,30,31}$.

Estudos recentes realizados em ratos anestesiados mostraram que a ativação dos neurônios do DMH resulta em ativação simpática para os leitos renal e cardíaco ${ }^{15,16,32}$. Adicionalmente, estudos em ratos acordados mostraram que a ativação do DMH produz aumento de frequência cardíaca e pressão arterial, que são acompanhados de aumento na atividade locomotora e nos níveis plasmáticos do hormônio adrenocorticotrópico $(\mathrm{ACTH})^{33}$ - este último considerado um importante sinalizador das respostas neuroendócrinas ao estresse ${ }^{34}$. A similaridade entre o efeito causado pela desinibição dos neurônios do DMH e a resposta ao estresse emocional permitiu sugerir que esta área seria fundamental na integração dessa resposta. Em conjunto, todos estes estudos indicam que o DMH parece ser um componente essencial das vias centrais mediadoras da resposta cardiovascular ao estresse emocional agudo.

\section{4- CONEXÕES E VIAS CENTRAIS DESCEN- DENTES: RVLM, RPa E PAG}

Embora todos esses achados tenham mostrado um papel de destaque para o DMH na geração da resposta cardiovascular ao estresse, com excessão de estudos anatômicos anteriores ${ }^{10,35,36}$, não haviam, até recentemente, estudos funcionais sobre as conexões centrais do DMH na geração da resposta cardiovascular produzida pela ativação deste núcleo. Esta seção descreverá, em ordem cronológica, os achados sobre as vias centrais descendentes mediadoras da resposta cardiovascular produzida pela ativação do DMH.

\section{1- Bulbo Rostroventrolateral}

Estudos anteriores mostraram, indiretamente, que o aumento da pressão arterial e frequência cardíaca produzidos pela ativação dos neurônios do DMH ocorriam através da ativação simpática ${ }^{37,38}$. No entanto, estudos anatômicos prévios também mostravam que o DMH contém poucos neurônios com projeção direta para a medula espinhal, de onde emergem os neurônios pré-ganglionares simpáticos ${ }^{10,35,36,39}$, indicando que a via simpatoexcitatória do $\mathrm{DMH}$ deveria conter uma ou mais conexões sinápticas com núcleos supramedulares. Baseando-se nessas observações, Fontes et al. ${ }^{15}$ mostraram que o aumento da pressão arterial e atividade simpática produzidos pela ativação dos neurônios do DMH foram reduzidos de maneira importante após inibição nos neurônios do bulbo rostroventrolateral (RVLM) - uma região que contém neurônios pré-motores simpáticos e crítica para o controle tônico e reflexo da pressão arterial ${ }^{40,41,42}$. Esses dados foram confirmados por Horiuchi et al. ${ }^{16}$, que mostraram que a frequência de disparo dos neurônios da RVLM aumentou cerca de 5 vezes após a microinjeção de BMI no DMH. No entanto, ao contrário da resposta pressora, a taquicardia produzida pela ativação dos neurônios do DMH não foi reduzida após inibição da RVLM ${ }^{15}$. Em um estudo subsequente, Cao et al. ${ }^{32}$ mostraram que a inibição dos neurônios da RVLM não altera o aumento na atividade simpática cardíaca produzida pela ativação do DMH. Estes resultados em conjunto sugeriram que os componentes vasomotor e cardíaco da resposta produzida pela ativação do DMH seriam mediados por núcleos relés diferentes ${ }^{15}$. Os neurônios do RVLM seriam um componente essencial das vias centrais mediadoras do aumento da atividade simpática para o leito vasomotor em resposta à ativação do DMH (Figura 3 , via $\mathrm{A}$ ). No entanto, as vias descendentes mediadoras da taquicardia - resposta marcante resultante da ativação do $\mathrm{DMH}$, parecia ser independente da RVLM.

\section{2- Rafe Pallidus}

Estudos posteriores ao de Fontes et al..$^{15}$ foram realizados no sentido de determinar qual via estaria envolvida na mediação da taquicardia produzida pela ativação do DMH. A rafe pallidus (RPa) foi considerada como um importante candidato, uma vez que: (1) esta região está entre as principais áreas do SNC que enviam projeções para a cadeia simpática na coluna intermediolateral da medula ${ }^{35}$ e; (2) desinibição dos neurônios da RPa resulta em aumento sustentado da atividade simpática para o nervo cardíaco e da frequência cardíaca em um padrão similar ao observado durante a ativação do $\mathrm{DMH}^{43,44}$. Estudos mostraram em ratos anestesiados que a taquicardia produzida pela ativação do DMH foi reduzida em aproximadamente 55\% após a inibição dos neurônios da Rafe Pallidus (Rpa) ${ }^{16,32,45}$. Adicionalmente, Zaretsky et al. ${ }^{46}$ mostraram que a microinjeção de muscimol na região da RP suprimiu a taquicardia associada com o estresse por jato de ar em ratos acordados. Em conjunto, esses estudos apontam a RPa como um possível relé sináptico na mediação da resposta taquicárdica produzida pelo DMH (Figura 3, via B). 


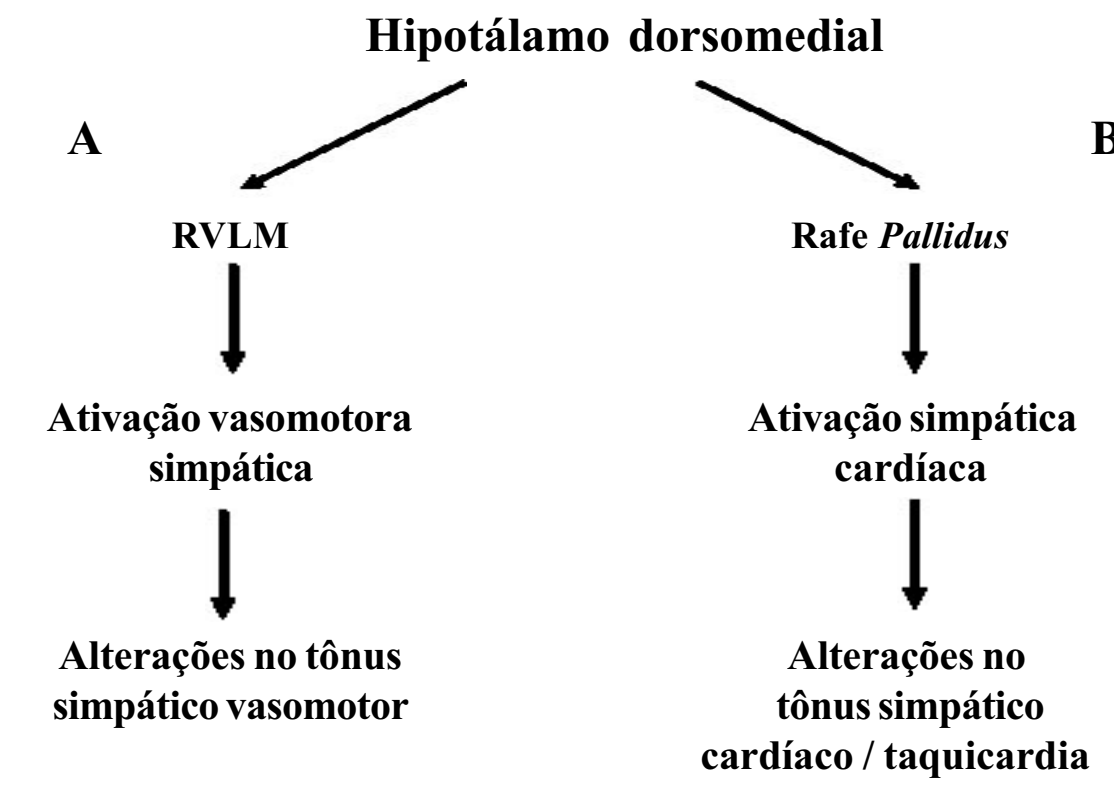

Figura 3: Esquema proposto a partir dos estudos que evidenciaram o papel da RVLM e rafe na mediação da resposta cardiovascular produzida pela ativação do DMH (ver texto para detalhes). RVLM, bulbo rostroventrolateral. Adaptado de Dampney et al., $2002^{55}$.

\section{3- Substância Cinzenta Periaquedutal}

Embora estudos anteriores mostrassem uma via funcional entre o DMH, RVLM e RPa ${ }^{15,16,45,46}$, estudos empregando técnicas de marcação neuronal anterógrada mostraram que o DMH envia projeções para outras regiões envolvidas no controle cardiovascular, como o núcleo do trato solitário (NTS) e a substância cinzenta periaquedutal (PAG) ${ }^{10,36}$. Desta forma, era possível considerar que as projeções do DMH para o RVLM e RP pudessem ser indiretas, incluindo relés sinápticos adicionais. A PAG foi considerada particularmente interessante, pois essa região recebe aferências do $\mathrm{DMH}^{10,36}$; e envia projeções para neurônios no RVLM e rafe $\mathrm{re}^{42,47,48}$. Estudos de Carrive et al. ${ }^{49}$ mostraram que a microinjeção de aminoácidos excitatórios na região lateral e dorsolateral da PAG produz um padrão coordenado de mudanças comportamentais e cardiovasculares, estes últimos caracterizados por hipertensão, taquicardia e alteração do fluxo sanguíneo para as diversas partes do corpo. Interessantemente, os efeitos observados após a ativação da PAG lateral e dorsolateral (1/dlPAG) se assemelham àqueles vistos após a ativação dos neurônios do DMH. Bandler et al. ${ }^{50}$ ja consideravam a PAG como um relé sináptico pelo qual o hipotálamo integrava os componentes somático e visceral do comportamento de defesa.
Baseando-se nestes estudos anteriores, Da Silva et. al ${ }^{51}$ consideraram o possível envolvimento dos neurônios da PAG na mediação da resposta cardiovascular produzida pela ativação do $\mathrm{DMH}^{14}$. Em experimentos realizados com o intuito de responder essa questão, Da Silva et al. observaram que o aumento na frequência cardíaca e pressão arterial produzidos pela ativação do DMH são reduzidos de forma importante, após a inibição dos neurônios da região lateral e dorsolateral da PAG (1/dlPAG) em ratos $\operatorname{acordados}^{14}$ (Figura 2). Em um estudo mais recente, Da Silva et al. mostraram que a resposta comportamental produzida pela ativação do DMH por bicuculina é similar à observada com a estimulação da 1/dlPAG por aminoácidos excitatórios ${ }^{51}$. Em conjunto, esses resultados indicam que a alteração de comportamento produzida pela ativação do DMH parece ser também dependente de uma conexão sináptica glutamatérgica com os neurônios da $\mathrm{PAG}^{51}$.

A PAG, assim como o DMH, não envia projeções diretas para a coluna intermediolateal, modulando as funções simpáticas provavelmente por vias indiretas $^{42}$. Considerando os estudos anteriores ${ }^{42,48}$, é possível sugerir que a PAG module essas funções através de neurônios prémotores simpáticos, como a RVLM, que recebe projeções anatômicas da PAG lateral ${ }^{42,48}$ e dos núcleos da rafe, que também rece- 
bem projeções da PAG lateral ${ }^{42}$. Dessa forma, é possível sugerir que, frente a uma situação de estresse, o $\mathrm{DMH}$ vai produzir as alterações cardiovasculares através de projeções excitatórias para a 1/dlPAG, que distribui o componente vasomotor para a RVLM; e o componente taquicárdico para os neurônios da rafe (Figura 4).

\section{5- DMH E A RESPOSTA CARDIOVASCULAR AO ESTRESSE: ABORDAGEMINTEGRATIVA}

Todas as observações anteriores indicam que o DMH pode ser uma região crítica na integração da resposta cardiovascular bem como de outras respostas em presença de estímulo emocional agudo, considerando respostas autonômicas ou não-autonômicas. Esta observação levanta a questão de como os estímulos emocionais poderiam ativar os neurônios do DMH. Nesse sentido o DMH estabelece conexões com a amígdala, estrutura classicamente envolvida nas respostas de medo condicionado ou amplamente conhecidas como memória emocional ${ }^{24,52}$. Ativação do núcleo basolateral da amígdala gera uma resposta similar àquela observada por um estressor agudo ${ }^{53} \mathrm{e}$ esta resposta é dependente de conexões sinápticas com o $\mathrm{DMH}^{54}$. Quando ativado, o $\mathrm{DMH}$ produziria uma resposta cardiovascular e comportamental integrada que seria distribuída pela PAG através da rafe e RVLM. A Figura 4 representa um modelo das conexões centrais envolvidas na mediação da resposta cardiovascular e comportamental em resposta à um estresse emocional agudo.

\section{6-PERSPECTIVAS}

Com base em experimentos em animais, Folkow ${ }^{9}$ mostrou que a ativação repetida da resposta cardiovascular ao estresse poderia resultar em hipertensão sustentada. Mais recentemente, estudos em humanos mostraram que agentes estressores de grande impacto produziram aumento sustentado na pressão arteri$\mathrm{al}^{6,7}$. Adicionalmente, Davies et al. ${ }^{8}$ demonstraram uma relação entre a desordem do pânico e a hipertensão. Uma vez que o DMH possui papel essencial na mediação das respostas cardiovasculares ao estresse, é possível que a ativação repetida do DMH através de situações de estresse possa ter um papel importante na gênese da hipertensão essencial, bem como de outras disfunções do sistema cardiovascular, como as arritmias cardíacas. Sem dúvida, estudos futuros sobre o possível papel do DMH na regulação a longo prazo da pressão arterial, assim como dos circuitos neurais envolvidos nessa regulação poderão trazer importantes esclarecimentos sobre os mecanismos envolvidos na fisiopatologia das doenças cardiovasculares.

\section{APOIO FINANCEIRO}

Fapemig, CNPq, CAPES. Da Silva Jr, LG e Villela, DC são bolsistas da CAPES. Menezes, RCA é bolsista do CNPq.

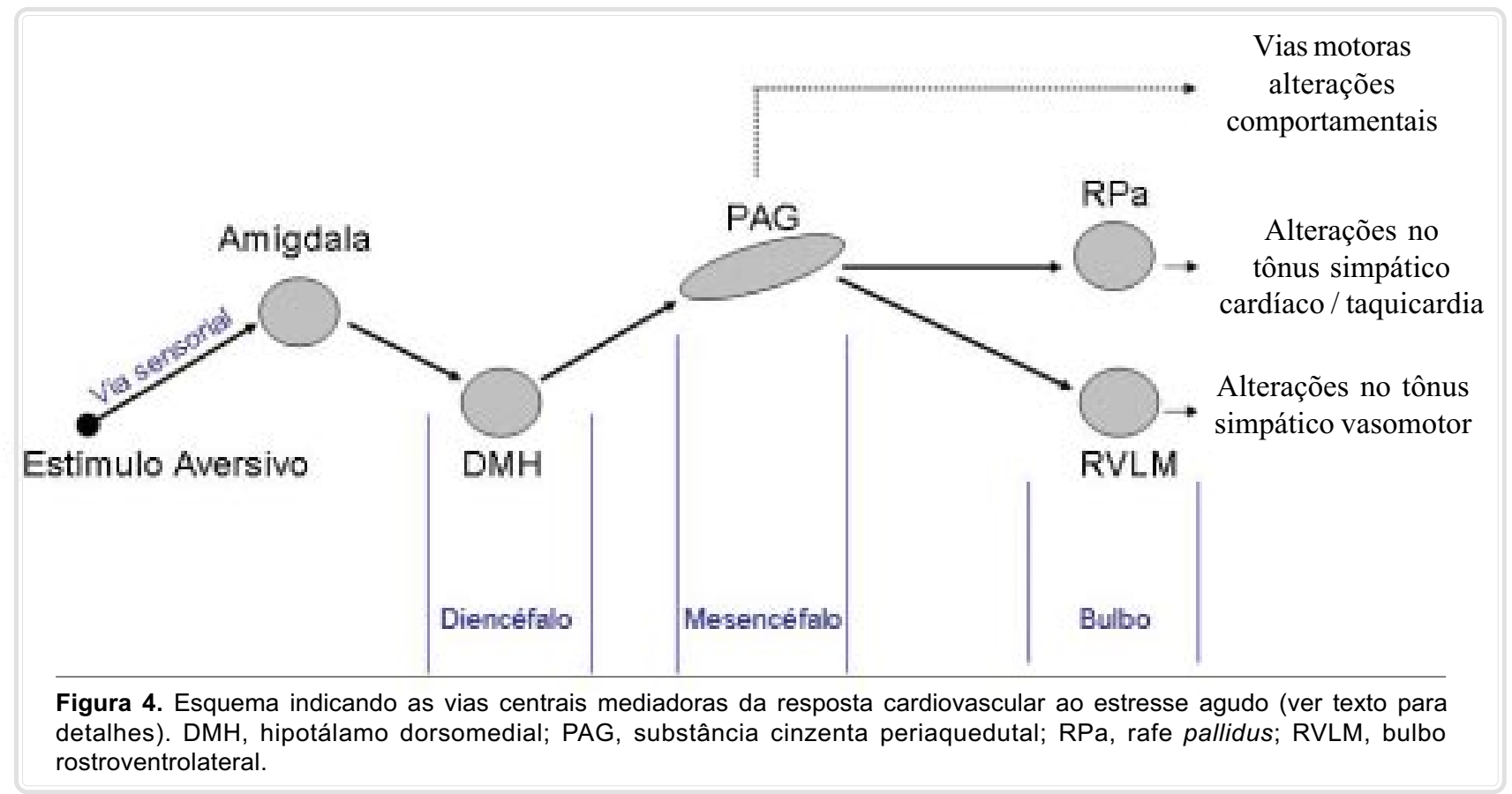


Fontes MAP, Menezes RCA, Villela DC, Silva Jr LG. The dorsomedial hypothalamus and the organization of the cardiovascular response to emotional stress: a functional perspective. Medicina (Ribeirão Preto) 2006; 39 (1): 13-20.

ABSTRACT: Emotional stress results in activation of specific pathways into central nervous system, which produces autonomic, behavioral and endocrine responses.

It is known that repetitive or continuous exposition to stress situations may result in various pathologic states, for example, arterial hypertension.

Classically, the hypothalamus plays an essential role in the integration of physiological responses to emotional stress. Recent studies demonstrate that a specific nucleus from hypothalamus, the dorsomedial hypothalamus $(\mathrm{DMH})$, is an essential component of central pathways that mediate the cardiovascular response to emotional stress. Inhibition of neurons in this area reduces the increases in heart rate and blood pressure in rats submitted to emotional stress paradigms. Conversely, pharmacological activation of DMH neurons evokes increases on heart rate, blood pressure, adrenocorticotrophic hormone, locomotor activity and sympathetic activity. The similarity of this response with that produced during emotional stress suggests that this area is crucial in the integration of the physiological responses to emotional stress.

The present review will discuss the central pathways used by $\mathrm{DMH}$ in the organization of the cardiovascular response to emotional stress.

Keywords: Dorsomedial Hypothalamus. Emotional Stress. Cardiovascular System. Descending Pathways.

\section{REFERÊNCIAS}

1 - Clark JD, Rager DR, Calpin JP. Animal well-being. II. Stress and distress. Lab Anim Sci 1997;47(6):571-9.

2 - Hilton SM Ways of viewing the central nervous control of the circulation—old and new. Brain Res 1975;87(2/3):213-9.

3 - Amiragova MG. Neurophysiological analysis of the development of endocrine and hypertensive reactions in prolonged emotional stress. Brain Res 1985;344(2):303-15.

4 - DiMicco JA, Samuels BC, Zaretskaia MV, Zaretsky DV. The dorsomedial hypothalamus and the response to stress: part renaissance, part revolution. Pharmacol Biochem Behav 2002;71(3):469-80.

5 - Leor J, Poole WK, Kloner RA. Sudden cardiac death triggered by an earthquake. N Engl J Med 1996;334(7):413-9.

6 - Gerin W, Chaplin W, Schwartz JE, Holland J, Alter R, Wheeler R, Duong D, Pickering TG. Sustained blood pressure increase after an acute stressor: the effects of the 11 September 2001 attack on the New York City World Trade Center. J Hypertens 2005;23(2):279-84.

7 - Parati G, Antonicelli R, Guazzarotti F, Paciaroni E, Mancia G. Cardiovascular effects of an earthquake: direct evidence by ambulatory blood pressure monitoring. Hypertension 2001;38(5):1093-5.

8 - Davies SJ, Ghahramani P, Jackson PR, Noble TW, Hardy PG, Hippisley-Cox J, Yeo WW, Ramsay LE. Association of panic disorder and panic attacks with hypertension. Am J Med 1999;107(4):310-6.

9 - Folkow B. Physiological aspects of primary hypertension. Physiol Rev 1982;62(2):347-504.

10 - Thompson RH, Canteras NS, Swanson LW. Organization of projections from the dorsomedial nucleus of the hypothalamus: a PHA-L study in the rat. J Comp Neurol 1996;376 (1): 143-73.
11 - Paxinos G, Watson C. The rat brain in stereotaxic coordinates. 2nd ed. New York: Academic Press; 1986.

12 - De Novellis V, Stotz-Potter EH, Morin SM, Rossi F, DiMicco JA. Hypothalamic sites mediating cardiovascular effects of microinjected bicuculline and EAAs in rats. Am J Physiol 1995;269(1 Pt 2): R131-40.

13 - Soltis RP, DiMicco JA. GABAA and excitatory amino acid receptors in dorsomedial hypothalamus and heart rate in rats. Am J Physiol 1991;260(1 Pt 2):R13-20.

14 - Da Silva LG, de Menezes RC, dos Santos RA, CampagnoleSantos MJ, Fontes MA. Role of periaqueductal gray on the cardiovascular response evoked by disinhibition of the dorsomedial hypothalamus. Brain Res 2003;984 (1-2): 206-14.

15 - Fontes MA, Tagawa T, Polson JW, Cavanagh SJ, Dampney RA. Descending pathways mediating cardiovascular response from dorsomedial hypothalamic nucleus. Am J Physiol Heart Circ Physiol 2001;280(6):H2891-901.

16 - Horiuchi J, McAllen RM, Allen AM, Killinger S, Fontes MA, Dampney RA. Descending vasomotor pathways from the dorsomedial hypothalamic nucleus: role of medullary raphe and RVLM. Am J Physiol Regul Integr Comp Physiol 2004;287(4):R824-32.

17 - Koutcherov Y, Mai JK, Paxinos G. Hypothalamus of the human fetus. J Chem Neuroanat 2003;26(4):253-70.

18 - Koutcherov Y, Mai JK, Ashwell KW, Paxinos G. Organisation of the human dorsomedial hypothalamic nucleus. Neuroreport 2004;15(1):107-11.

19 - Freedman RR, lanni P, Ettedgui E, Puthezhath N. Ambulatory monitoring of panic disorder. Arch Gen Psychiatry 1985; 42 (3):244-8.

20 - Stein MB, Tancer ME, Uhde TW. Heart rate and plasma norepinephrine responsivity to orthostatic challenge in anxiety disorders. Comparison of patients with panic disorder and social phobia and normal control subjects. Arch Gen Psychiatry 1992;49(4):311-7. 
21 - Rechlin T, Weis M, Spitzer A, Kaschka WP. Are affective disorders associated with alterations of heart rate variability? J Affect Disord 1994;32(4):271-5.

22 - Shekhar A, Keim SR, Simon JR, McBride WJ. Dorsomedial hypothalamic GABA dysfunction produces physiological arousal following sodium lactate infusions. Pharmacol Biochem Behav 1996;55(2):249-56.

23 - Randall DC, Hasson DM. Cardiac arrhythmias in the monkey during classically conditioned fear and excitement. Pavlov J Biol Sci 1981;16(2):97-107.

24 - LeDoux JE. Emotion circuits in the brain. Annu Rev Neurosci 2000;23:155-84.

25 - DiMicco JA, Stotz-Potter EH, Monroe AJ, Morin SM. Role of the dorsomedial hypothalamus in the cardiovascular response to stress. Clin Exp Pharmacol Physiol 1996;23(2):171-6.

26 - Brown DA, Higgins AJ, March S, Smart TG. Actions of GABA on mammalian neurones, axons and nerve terminals. New York: Raven; 1981.

27 - Stotz-Potter EH, Willis LR, DiMicco JA. Muscimol acts in dorsomedial but not paraventricular hypothalamic nucleus to suppress cardiovascular effects of stress. J Neurosci 1996;16(3):1173-9.

28 - DiMicco JA, Abshire VM, Hankins KD, Sample RH, Wible JH, Jr. Microinjection of GABA antagonists into posterior hypothalamus elevates heart rate in anesthetized rats. Neuropharmacology 1986;25(9):1063-6.

29 - Soltis RP, DiMicco JA. Interaction of hypothalamic GABAA and excitatory amino acid receptors controlling heart rate in rats. Am J Physiol 1991;261(2 Pt 2):R427-33.

30 - Shekhar A. GABA receptors in the region of the dorsomedial hypothalamus of rats regulate anxiety in the elevated plusmaze test. I. Behavioral measures. Brain Res 1993; 627 (1): 9-16.

31 - Shekhar A, Sims LS, Bowsher RR. GABA receptors in the region of the dorsomedial hypothalamus of rats regulate anxiety in the elevated plus-maze test. II. Physiological measures. Brain Res 1993;627(1):17-24.

32 - Cao WH, Fan W, Morrison SF. Medullary pathways mediating specific sympathetic responses to activation of dorsomedial hypothalamus. Neuroscience 2004;126(1):229-40.

33 - Bailey TW, Dimicco JA. Chemical stimulation of the dorsomedial hypothalamus elevates plasma ACTH in conscious rats. Am J Physiol Regul Integr Comp Physiol 2001;280(1):R8-15.

34 - Pacak K, Palkovits M. Stressor specificity of central neuroendocrine responses: implications for stress-related disorders. Endocr Rev 2001;22(4):502-48.

35 - Hosoya Y, Ito R, Kohno K. The topographical organization of neurons in the dorsal hypothalamic area that project to the spinal cord or to the nucleus raphe pallidus in the rat. Exp Brain Res 1987;66(3):500-6.

36 - ter Horst GJ, Luiten PG. The projections of the dorsomedial hypothalamic nucleus in the rat. Brain Res Bull 1986;16(2):231-48.

37 - Wible JH, Jr, DiMicco JA, Luft FC. Hypothalamic GABA and sympathetic regulation in spontaneously hypertensive rats. Hypertension 1989;14(6):623-8.

38 - Wible JH, Jr., Luft FC, DiMicco JA. Hypothalamic GABA suppresses sympathetic outflow to the cardiovascular system. Am J Physiol 1988;254(4 Pt 2):R680-7.

39 - Abshire VM, Hankins KD, Roehr KE, DiMicco JA. Injection of L-allylglycine into the posterior hypothalamus in rats causes decreases in local GABA which correlate with increases in heart rate. Neuropharmacology 1988;27(11):1171-7.
40 - Dampney RA, Tagawa T, Horiuchi J, Potts PD, Fontes M, Polson JW. What drives the tonic activity of presympathetic neurons in the rostral ventrolateral medulla? Clin Exp Pharmacol Physiol 2000;27(12):1049-53.

41 - Dampney RA. Functional organization of central pathways regulating the cardiovascular system. Physiol Rev 1994;74(2):323-64.

42 - Farkas E, Jansen AS, Loewy AD. Periaqueductal gray matter input to cardiac-related sympathetic premotor neurons. Brain Res 1998;792(2):179-92.

43 - Cao WH, Morrison SF. Disinhibition of rostral raphe pallidus neurons increases cardiac sympathetic nerve activity and heart rate. Brain Res 2003;980(1):1-10.

44 - Morrison SF, Sved AF, Passerin AM. GABA-mediated inhibition of raphe pallidus neurons regulates sympathetic outflow to brown adipose tissue. Am J Physiol 1999; 276 (2 Pt 2):R290-7.

45 - Samuels BC, Zaretsky DV, DiMicco JA. Tachycardia evoked by disinhibition of the dorsomedial hypothalamus in rats is mediated through medullary raphe. J Physiol 2002; 538 (Pt 3): 941-6.

46 - Zaretsky DV, Zaretskaia MV, Samuels BC, Cluxton LK, DiMicco JA. Microinjection of muscimol into raphe pallidus suppresses tachycardia associated with air stress in conscious rats. $J$ Physiol 2003; 546 (Pt 1): 243-50.

47 - Carrive P, Bandler R, Dampney RA. Anatomical evidence that hypertension associated with the defence reaction in the cat is mediated by a direct projection from a restricted portion of the midbrain periaqueductal grey to the subretrofacial nucleus of the medulla. Brain Res 1988;460(2):339-45.

48 - Hudson PM, Lumb BM. Neurones in the midbrain periaqueductal grey send collateral projections to nucleus raphe magnus and the rostral ventrolateral medulla in the rat. Brain Res 1996;733(1):138-41.

49 - Carrive P, Dampney RA, Bandler R. Excitation of neurones in a restricted portion of the midbrain periaqueductal grey elicits both behavioural and cardiovascular components of the defence reaction in the unanaesthetised decerebrate cat. Neurosci Lett 1987;81(3):273-8.

50 - Bandler RJ Jr, Chi CC, Flynn JP. Biting attack elicited by stimulation of the ventral midbrain tegmentum of cats. Science 1972;177(46):364-6.

51 - Da Silva LG, Menezes RCA, Villela DC, Fontes MAP. Excitatory amino acid receptors in the periaqueductal gray mediate the cardiovascular response evoked by activation of the dorsomedial hypothalamic neurons. Neuroscience. In: Press 2006.

52 - LeDoux J. The emotional brain, fear, and the amygdala. Cell Mol Neurobiol 2003;23(4-5):727-38

53 - Sanders SK, Shekhar A. Blockade of GABAA receptors in the region of the anterior basolateral amygdala of rats elicits increases in heart rate and blood pressure. Brain Res 1991;567(1):101-10.

54 - Soltis RP, Cook JC, Gregg AE, Stratton JM, Flickinger KA. EAA receptors in the dorsomedial hypothalamic area mediate the cardiovascular response to activation of the amygdala. Am J Physiol 1998;275(2 Pt 2):R624-31.

55 - Dampney RAL, Coleman MJ, Fontes MAP, Hirooka Y, Horiuchi J, Li Y-W, Polson JW, Potts PD, Tagawa T. Central mechanisms underlying short and long-term regulation of the cardiovascular system. Clin Exp Pharmacol Physiol 2002; 29:261-8 